\title{
Liquid metal machine is evolving to soft robotics
}

\author{
LIU Jing ${ }^{1,2 *}$ \\ ${ }^{1}$ Technical Institute of Physics and Chemistry, Chinese Academy of Sciences, Beijing 100190, China; \\ ${ }^{2}$ Department of Biomedical Engineering, School of Medicine, Tsinghua University, Beijing 100084, China
}

Received September 9, 2016; accepted October 10, 2016; published online October 12, 2016

\begin{abstract}
Making soft robots that can flexibly transform among different morphologies has long been a dream in both science and engineering areas. With outstanding versatile capabilities, liquid metals are opening breakthrough strategies for molding future smart soft robots that had never been anticipated before or hardly achievable by a rigid metal or conventional materials $[1,2]$. All the evidences collected so far pointed out that, liquid metal machine is evolving via a rather quick way.

The latest discoveries on a group of very fundamental phenomena [3-7] of liquid metals further strengthen this endeavor. Unlike former trials on pure liquid metal, the new findings [3-6] revealed that hybrid structures with both liquid and solid elements together would enable more complicated functions. As it reveals [3], when contacting a copper wire to the liquid metal fueled with aluminum, the wire will be swallowed and then moves back and forth, just like a violin bow. Such oscillating motion could be regulated and speeded up by touching a steel needle on the liquid metal surface. This autonomous oscillator can be developed as a switch element in periodically controlling fluidic, electrical, mechanical and optical functions.

In fact, combining solid components with liquid metal can produce more machine roles. For example, contacting nickel particles to sub-millimeter EGaIn droplet in $\mathrm{NaOH}$ solution would induce intermittent jumping of the metal droplet [4]. The reason comes from the electron discharge effect due to point contact between liquid metal and solid metal particles in electrolyte. It is the short circuit effect that significantly enhances the near-surface electric field at the particle tips which then causes electric breakdown of the electrolyte and thus drastic hydrogen generation leading to strong propelling force.
\end{abstract}

\footnotetext{
*Corresponding author (email: jliu@ mail.ipc.ac.cn)
}

Alternatively, if intentionally electroplating more other materials like a magnetic Ni layer on the liquid metal, the autonomous $\mathrm{Ni} / \mathrm{Al} / \mathrm{EGaIn}$ machine fueled with aluminum could be manipulated by external magnet or electric field which can realize diverse motion control such as steering, changing direction, start, and stop [5]. Through further coating with soft biomedical hydrogel, the liquid metal motor can even be utilized to deliver drugs.

Moreover, liquid metal can be integrated with solid structure to construct various machines. In this side, metal droplet was demonstrated to be used as "wheels" to drive 3D printed vehicle or boat [6]. The hybrid machine thus made adapts to multiple electrolytes especially $\mathrm{NaOH}$ solution. Under variable electrical voltages and configurations, the vehicle can be controlled to achieve various progressing, steering, carrying freight and more locomotion states.

In earlier efforts, several pivotal findings on shape changeable liquid metal are on the electrically induced liquid metal transformers. It was disclosed that through applying electrical field on the liquid metals immersed in or sprayed with water, very unusual phenomena would occur such as transformation from a large sized metal film into a tiny sphere, quick mergences of separate metal droplets, controlled self-rotation and planar locomotion of liquid metal objects or machines in free space or confined structures [1]. Reversible behaviors were also achieved via the synthetically chemical-electrical mechanism (SCHEME) [2], which combines chemical dissolution and electrochemical oxidation together. Except for that, facing the big challenge in controlling the shape of liquid metal due to its extremely high surface tension, a latest work [7] opens a way for easily manipulating liquid metal through introducing graphite as special functional substrate. Due to interactions on the interface, liquid metal (LM) in alkaline electrolyte can be fixed into any desired stable shapes like flat, dull and sharp 
angle. Besides, unique transformations and worm-like antigravity upslope LM locomotion were also made possible.

Without any doubt, it now can be conceived that, liquid metal machines are evolving towards sophisticated soft robots. However, it should also be pointed out that, if only relying on external field, the liquid metal machines can be termed at most as "shape changeable" machine which is still far from biologically featured robot. Fortunately, the evolution course was significantly advanced owing to discovery of the extraordinary self-powered liquid metal effect [8]. Such long lasting and quickly running soft machines work just like a biomimetic mollusk since they closely resemble the nature by "eating" aluminum as "food", and can change shape by closely conforming to the geometrical space they voyage in. This new generation machine owns the capabilities of autonomous convergence or divergence during chasing [9] which are hardly achievable otherwise by conventional machines, even biological systems.

Also unexpectedly, it was found that if injecting a large liquid metal machine fueled with aluminum into aqueous solution, tremendous tiny self-running motors can be spontaneously generated which run here and there as a swarm of soft machines [10]. If putting them into confined space, they can get back to the original one. Over the process, each tiny machine just keeps its running, colliding, bouncing, coalescence or adhesion states until finally assembles into a single large machine. In this sense, liquid metal machine can work as either a large size robot, partial running elements or just divided spontaneously running motors. It is for this reason, we have defined a new robot concept termed as "Transient State Machine" [11]. That means, such machine could dynamically display with different geometries in single or swarm style, small or large size, assembling or interaction etc. If one wishes to control the liquid metal motors, electrical or magnetic field could work well to speed up, stop the motors or regulate their running directions $[12,13]$.

Overall, the finding of artificial liquid metal mollusk opens an exciting platform for designing future soft robot, microfluidic systems, and may eventually lead to the envisioned dynamically reconfigurable intelligent soft robots or machines. Aiming for this goal, we had ever initiated a project named as SMILE (Soft Machine based on Intelligence, Liquid-metal and Electronics). Clearly, all these machine or robot styles are based on liquid metal's soft, fluidic and multi-physical and chemical capabilities.

Lastly, if taking a deep thinking about nature, it would be interesting to note that all life systems including both plants and animals own such a basic feature: "soft". Liquid metal machine perfectly mimics this power in nature. It is "soft" that distinguishes the biology or liquid metal machine from the abiotic matter and enables a wonderful vibrant world. In this sense, we can even define "soft" as another dimension apart from the classical dimensional concepts regarding time and geometries. This is because with soft property inside, material can change shape, dimensions and undertake tough task. For example, a liquid metal wire packed in plas- tic pipe could be easily stretched or separated. While for a liquid metal sphere immersed in aqueous solution, external fields such as electricity would be able to induce its interior circulation. In that case, although size, weight even shape does not vary, the dimension of the object has already been changed. Therefore, "soft" serves well to characterize the new dimension. And softness spanning from 0 to 1 could provide more quantifications on such dimensional behaviour. For example, liquid metal may stay in completely soft state which can reorganize into any different shapes. While for a perfect rigid material, its softness is just zero. It means that such object could not change shape unless machined. For soft robots composed of liquid metal and other materials, a desired softness in 0-1 can be obtained. Thus, there is plenty of space in innovating liquid metal and aligned materials to compose advanced soft robots.

In summary, with soft capability and fluidic feature inside, the liquid metal is rather promising in evolving into further complicated machine styles whose ultimate goal is biology like robots. The next step for advancing liquid metal machine is to just fully simulate nature. Meanwhile, some newly emerging liquid metal 3D printing methods will also aid for such machine fabrications [14, 15]. It is expected that a wonderful world of liquid metal soft robots will come in the near future.

1 Sheng L, Zhang J, Liu J. Diverse transformations of liquid metals between different morphologies. Adv Mater, 2014, 26: 6036-6042

2 Zhang J, Sheng L, Liu J. Synthetically chemical-electrical mechanism for controlling large scale reversible deformation of liquid metal objects. Sci Rep, 2014, 4: 7116

3 Yuan B, Wang L, Yang X, et al. Liquid metal machine triggered violin-like wire oscillator. Adv Sci, 2016, doi: 10.1002/advs.201600212

4 Tang J B, Wang J, Liu J, et al. Jumping liquid metal droplet in electrolyte triggered by solid metal particles. App Phys Lett, 2016, 108: 223901

5 Zhang J, Guo R, Liu J. Self-propelled liquid metal motors steered by magnetic or electrical field for drug delivery. J Mater Chem B, 2016, 4: 5349-5357

6 Yao Y, Liu J. Liquid metal wheeled small vehicle for cargo delivery. RSC Adv, 2016, 6: 56482-56488

$7 \mathrm{Hu}$ L, Wang L, Ding Y, et al. Manipulation of liquid metals on a graphite surface. Adv Mater, 2016, doi: 10.1002/adma.201601639

8 Zhang J, Yao Y, Sheng L, et al. Self-fueled biomimetic liquid metal mollusk. Adv Mater, 2015, 27: 2648-2655

9 Zhang J, Yao Y, Liu J. Autonomous convergence and divergence of the self-powered soft liquid metal vehicles. Sci Bull, 2015, 60: 943-951

10 Yuan B, Tan S, Zhou Y X, et al. Self-powered macroscopic Brownian motion of spontaneously running liquid metal motors. Sci Bull, 2015, 60: 1203-1210

11 Sheng L, He Z, Yao Y, et al. Transient state machine enabled from the colliding and coalescence of a swarm of autonomously running liquid metal motors. Small, 2015, 11: 5253-5261

12 Tan S C, Yuan B, Liu J. Electrical method to control the running direction and speed of self-powered tiny liquid metal motors. Proc Roy Soc A, 2015, 471, doi: 10.1098/rspa.2015.0297

13 Tan S C, Gui H, Yuan B, et al. Magnetic trap effect to restrict motion of self-powered tiny liquid metal motors. App Phys Lett, 2015, 107: 071904

14 Wang L, Liu J. Liquid phase 3D printing for quickly manufacturing conductive metal objects with low melting point alloy ink. Sci China Tech Sci, 2014, 57: 1721-1728

15 Wang L, Liu J. Compatible hybrid 3D printing of metal and nonmetal inks for direct manufacture of end functional devices. Sci China Tech Sci, 2014, 57: 2089-2095 